\title{
Predicted Suppression of the Superconducting Transition of New High-Pressure Yttrium Phases with Increasing Pressure from First-Principles Calculations
}

\author{
Yue Chen (陈粤), ${ }^{*}$ Qing-Miao Hu (胡青苗), and Rui Yang (杨锐) \\ Shenyang National Laboratory for Materials Science, Institute of Metal Research, Chinese Academy of Sciences, \\ 72 Wenhua Road, Shenyang 110016, China \\ (Received 20 February 2012; published 10 October 2012)
}

\begin{abstract}
Structure searches for new high-pressure phases of Y metal have been performed by using evolutionary algorithms in conjunction with a first-principles, pseudopotential plane-wave method based on density functional theory. The $o F 16-F d d d$ and $h P 3-P 3_{1} 21$ phases are predicted to be energetically favorable at pressures over $97 \mathrm{GPa}$. These two phases are shown to be dynamically stable by computing their phonon dispersions. We thus propose that $o F 16-F d d d$ and $h P 3-P 3_{1} 21$ are the most probable crystal structures $\mathrm{Y}$ may take in the 97-206 GPa range. The superconducting critical temperatures $\left(T_{c}\right)$ of the new phases are estimated using the Allen-Dynes formula. The $T_{c}$ is predicted to decrease with increasing pressure over about $100 \mathrm{GPa}$, in sharp contrast to its observed monotonic increase under lower pressure. The electronic origins of the stabilities of the proposed high-pressure phases have also been investigated.
\end{abstract}

Experimental determination of the crystal structures of materials becomes difficult under extremely high pressure. Fortunately, recent developments in the methods for theoretical predictions of crystal structures provide an effective way to search for novel high-pressure phases. A number of successful applications of these methods which include the $a b$ initio random structure searching [1-6] and evolutionary approaches [7-11] have been reported recently. As an example, sodium was predicted to take the $h P 4$ structure under high pressure by Ma et al. [7] who employed an evolutionary approach. The $h P 4$ phase has then been found to be responsible for the transformation of $\mathrm{Na}$ into an optically transparent phase at about $200 \mathrm{GPa}$. The successful applications of the structure searching approaches show their capabilities of predicting complex configurations given only the compositions of the system.

A large number of elements and compounds undergo superconducting transitions under pressure, in particular, the critical temperatures $T_{c}$ may increase with hydrostatic pressures. Therefore, the predictions of the crystal structures of high-pressure phases become invaluable in searching for high $T_{c}$ superconductors. Yttrium has recently been reported to exhibit superconductivity at 17 and $20 \mathrm{~K}$ at pressures of 89 and $115 \mathrm{GPa}$, respectively, which qualifies it (together with $\mathrm{Li}[12]$ ) as the highest $T_{c}$ elemental superconductor [13-15].

Within the framework of routine density functional theory (DFT), the current authors investigated the structural phase transitions of $\mathrm{Y}$ with pressure up to $300 \mathrm{GPa}$ in a recent publication [16]. The structural transition pressures with regard to, hexagonal close-packed (hcp) $\rightarrow$ samarium-type (Sm-type) $\rightarrow$ double hexagonal closepacked (dhcp) $\rightarrow$ distorted face-centered cubic (dfcc), was estimated to be 0,7 and $51 \mathrm{GPa}$, respectively. In addition, a new phase $h P 3-P 6_{2} 22$ (the Pearson symbol and space group), which had never been observed experimentally, was predicted to be energetically preferable over about $100 \mathrm{GPa}$. However, the $h P 3-P 6_{2} 22$ phase is unlikely to be observed at a pressure lower than $206 \mathrm{GPa}$ due to the existence of imaginary phonon frequencies. Large enthalpy differences between the $h P 3-P 6_{2} 22$ and dfcc phases in the pressure range of 100-206 GPa imply the possible existence of yet unknown phases.

Since the crystal structure of Y in the 100-206 GPa range is of great importance for understanding its extraordinary superconducting behaviors, in this Letter, we perform theoretical searches for the new phases of $\mathrm{Y}$ at $140 \mathrm{GPa}$ using evolutionary algorithms (EAs) and a firstprinciples method based on DFT. A group of energetically preferable crystal structures found in the evolutionary searches are then investigated in detail and compared with the experimentally observed phases with pressure up to $300 \mathrm{GPa}$. It is found that, considering the accuracy of DFT calculations, the oF 16-Fddd, $h P 3-P 3_{1} 21$ and $h P 3-P 6_{2} 22$ phases become indistinguishable in enthalpy over about $100 \mathrm{GPa}$. Moreover, contrary to the $h P 3-P 6_{2} 22$ phase showing imaginary phonon frequencies at $97 \mathrm{GPa}$ [16], the $o F 16-F d d d$ and $h P 3-P 3_{1} 21$ phases are dynamically stable, which makes them the most probable crystal structures Y may take over about $100 \mathrm{GPa}$. Based on our newly proposed crystal structures, the Eliashberg functions $\alpha^{2} F(\omega)$ and the electron-phonon (e-ph) coupling constants $\lambda$ are calculated to analyze the superconducting features of $\mathrm{Y}$ under high pressure. The projected band structures, density of states (DOS), and charge density differences of the $o F 16-F d d d$ and $h P 3-P 3_{1} 21$ phases at 0 and $97 \mathrm{GPa}$ are then compared to investigate the electronic origins of their stabilities at high pressure.

We have performed evolutionary searches for the energetically preferable crystal structures at $T=0 \mathrm{~K}$ and 
$P=140 \mathrm{GPa}$ using USPEX [17-19] in conjunction with VASP [20,21], which enable us to search for the global minimum of the enthalpy. Unit cells with 8, 10, and 12 atoms are used for the EA structure searches. For local crystal structure relaxations, projector augmented wave pseudopotentials [22] are applied, and the generalized gradient approximation [23] is adopted to describe the electronic exchange-correlation potential. The semicore $4 s$ and $4 p$ orbitals of $\mathrm{Y}$ have been treated as valence states. We apply a plane-wave energy cutoff of $480 \mathrm{eV}$ and dense $\left(2 \pi \times 0.025 \AA^{-1}\right.$ resolution) Monkhorst-Pack $k$ meshes [24] to ensure that the enthalpy differences converge to typically 1-2 meV/atom. Cell shapes and atomic coordinates are relaxed with the constraints of constant volumes before the enthalpies are calculated as functions of pressure. Phonon dispersions and $e$-ph couplings are computed within the density functional perturbation theory (DFPT) using the QUANTUM-ESPRESSO package $[25,26]$.

The unit cells of the energetically preferable crystal structures produced by EA structure searches at $140 \mathrm{GPa}$ are shown in the top panel of Fig. 1. The $o C 8-C 222_{1}$, $o F 8-F d d d, o F 16-F d d d$, and $h P 3-P 3_{1} 21$ structures have four, two, four, and three atoms in their primitive cells, respectively. The enthalpies of $\mathrm{Y}$ in these four crystal
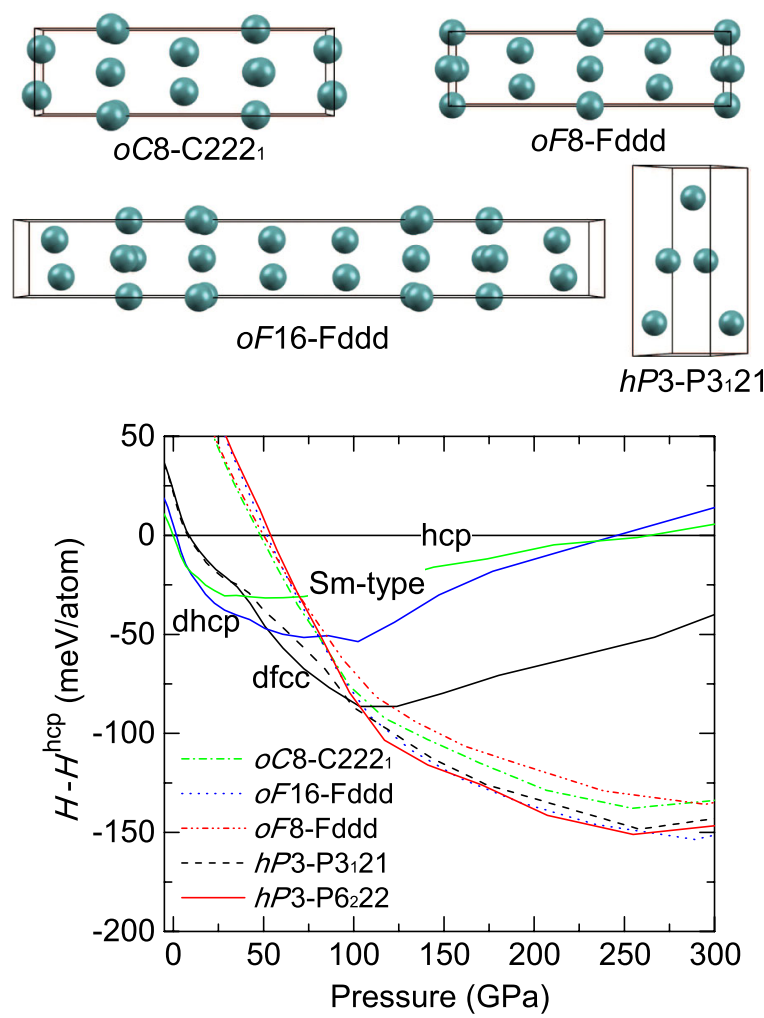

FIG. 1 (color online). Top: The unit cells of the energetically preferable phases generated by first-principles evolutionary searches at $140 \mathrm{GPa}$. Bottom: The enthalpy differences (relative to hcp) of $\mathrm{Y}$ in competing crystal structures as functions of pressure. structures are computed as functions of pressure and compared with the experimentally observed phases as well as the theoretically predicted $h P 3-P 6_{2} 22$ [16]. It is worth noting that both of the dfcc and $h P 3-P 3_{1} 21$ phases transform to fcc during structural relaxations and show identical enthalpies at pressures below about $35 \mathrm{GPa}$. In addition, the enthalpies of the $o F 16-F d d d, h P 3-P 3_{1} 21$, and $h P 3-P 6_{2} 22$ phases become indistinguishable within the accuracy of typical DFT calculations and the lowest among all the competing phases at pressures above $97 \mathrm{GPa}$. In our previous publication [16], we pointed out that the $h P 3-P 6_{2} 22$ phase is dynamically unstable below about $206 \mathrm{GPa}$ due to the imaginary phonon frequencies; therefore, $o F 16-F d d d$ and $h P 3-P 3_{1} 21$ produced by EA structure searches become the candidate crystal structures of $\mathrm{Y}$ in the 97-206 GPa pressure range. Nevertheless, the $o C 8-C 222_{1}$ and $o F 8-F d d d$ phases are unlikely to be observed experimentally due to their relatively high enthalpies throughout the whole pressure range that we have considered.

The predicted lattice constants and atomic coordinates of the $o F 16-F d d d, h P 3-P 3_{1} 21$, and $h P 3-P 6_{2} 22$ phases at $97 \mathrm{GPa}$ are summarized in Table I. Not surprisingly, the lattice constants of the $h P 3-P 3_{1} 21$ and $h P 3-P 6_{2} 22$ phases are very close to each other due to the similar atomic arrangements in their unit cells. It is also found that $h P 3-P 3_{1} 21$ transforms to $h P 3-P 6_{2} 22$ with the atomic coordinates taking $(0.50,0.50,0.83)$ during structural relaxations at pressures above approximately $200 \mathrm{GPa}$. Under the perturbation of the imaginary phonon mode shown in our previous publication [16], $h P 3-P 6_{2} 22$ relaxes to $h P 3-P 3_{1} 21$ (or its enantiomorph $h P 3-P 3_{2} 21$ ) below about $200 \mathrm{GPa}$.

To be observed experimentally under high pressure, the $o F 16-F d d d$ and $h P 3-P 3_{1} 21$ phases have to be dynamically stable. To clarify this issue, phonon dispersions along the high-symmetry directions of the Brillouin zones of these two phases at $97 \mathrm{GPa}$ are computed and shown in the top panels of Fig. 2. Contrary to the $h P 3-P 6_{2} 22$ phase which exhibits imaginary phonon frequencies throughout the Brillouin zone at $97 \mathrm{GPa}$ (see Fig. 5 of Ref. [16]), no soft modes are found for the $o F 16-F d d d$ and $h P 3-P 3_{1} 21$ phases. Thus, we propose that $o F 16-F d d d$ and $h P 3-P 3_{1} 21$ are the most probable crystal structures $\mathrm{Y}$ may take under pressures above approximately $100 \mathrm{GPa}$, where the

TABLE I. Predicted lattice constants and atomic coordinates, as referred to the conventional unit cells, of the $o F 16-F d d d$, $h P 3-P 3_{1} 21$ and $h P 3-P 6_{2} 22$ phases at $97 \mathrm{GPa}$.

\begin{tabular}{lccrc}
\hline \hline Structure & $\mathrm{a}(\AA)$ & $\mathrm{b}$ & \multicolumn{1}{c}{$\mathrm{c}$} & Fractional coordinate \\
\hline$o F 16-F d d d$ & 4.81 & 2.83 & 17.70 & $(0.25,0.25,0.56)$ \\
$h P 3-P 3_{1} 21$ & 2.79 & 2.79 & 6.71 & $(0.56,0.44,0.83)$ \\
$h P 3-P 6_{2} 22$ & 2.81 & 2.81 & 6.60 & $(0.50,0.50,0.83)$ \\
\hline \hline
\end{tabular}




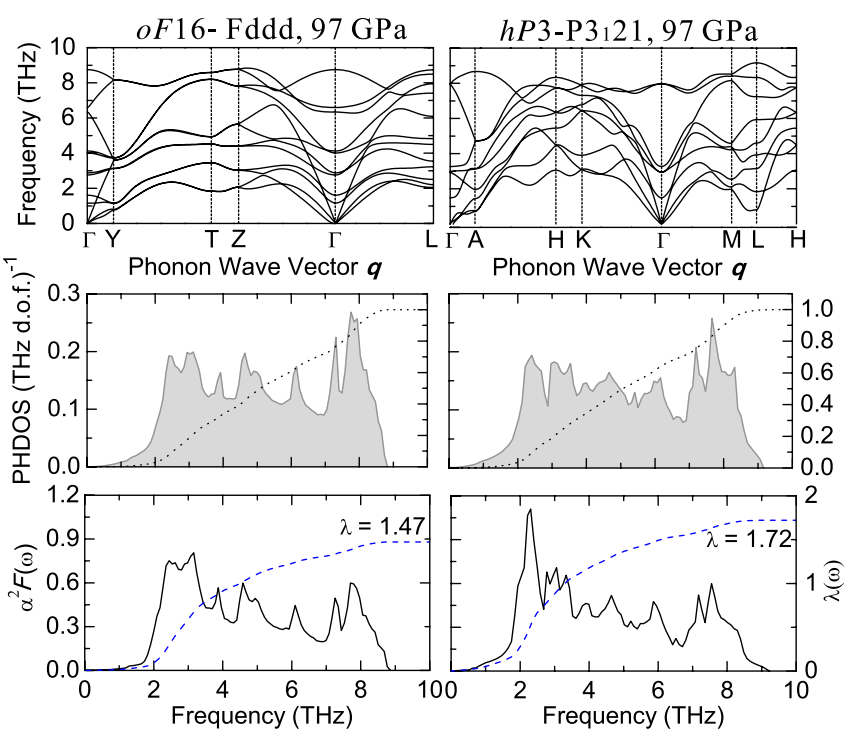

FIG. 2 (color online). The phonon dispersions (top), PHDOS (middle), and Eliashberg functions $\alpha^{2} F(\omega)$ (bottom) of the $o F 16-F d d d$ and $h P 3-P 3_{1} 21$ phases at $97 \mathrm{GPa}$. The integrals of PHDOS (dotted lines) and the $e$-ph coupling constants $\lambda$ (dashed lines) are referenced to the right axes.

experimentally observed dfcc phase becomes energetically unfavorable.

Hamlin et al. have recently reported that the superconducting critical temperature $T_{c}$ of Y increased monotonically with pressure and reached a record value of $20 \mathrm{~K}$ at $115 \mathrm{GPa}$ [13]. Based on our newly proposed high-pressure phases of Y, we are now able to theoretically estimate the $T_{c}$ up to a higher pressure.

The phonon density of states (PHDOS), Eliashberg functions $\alpha^{2} F(\omega)$, and $e$-ph coupling constants $\lambda$ of the $o F 16-F d d d$ and $h P 3-P 3_{1} 21$ phases at $97 \mathrm{GPa}$ are computed using fine $k$ and $q$ meshes (see the middle and bottom panels of Fig. 2). The Eliashberg functions integrate to large $\lambda$ of 1.47 and 1.72 for the $o F 16-F d d d$ and $h P 3-P 3_{1} 21$ phases, respectively, with over half of the values contributed from the phonon modes in the range of 2-4 THz. By assigning $\mu^{*}$ with a typical value in the $0.10-0.15$ range, we have evaluated $T_{c}$ using Allen-Dynes formula $[27,28]$. Both of the $o F 16-F d d d$ and $h P 3-P 3_{1} 21$ phases are estimated to exhibit $T_{c}$ in the range of $16-19 \mathrm{~K}$ at $97 \mathrm{GPa}$.

By using the same scheme, the $T_{c}$ of different highpressure phases of $\mathrm{Y}$ is calculated and compared with available experimental values in Fig. 3. For the dhcp and dfcc phases, the theoretical $T_{c}$ increases with pressure, in good agreement with experimental measurements. In sharp contrast to the behavior of $T_{c}$ against pressures below $100 \mathrm{GPa}$, the $T_{c}$ of the newly predicted phases (oF16- $F d d d$ and $\left.h P 3-P 3_{1} 21\right)$ at higher pressure decreases with pressure. In other words, a higher $T_{c}$ may not be observed in experiments by further increasing the pressure

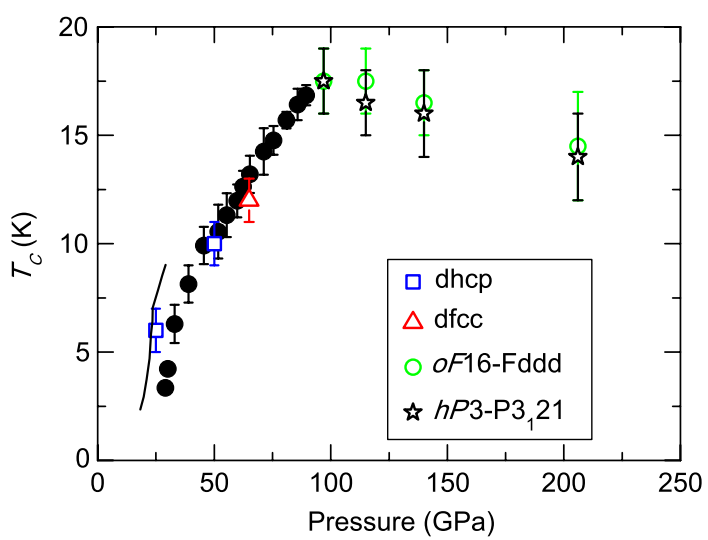

FIG. 3 (color online). The theoretically estimated superconducting critical temperatures $T_{c}$ (open symbols) of different ground-state structures of $\mathrm{Y}$ under high pressures. The solid circles and line denote the experimental values (see Fig. 2 of Ref. [13]).

beyond about $100 \mathrm{GPa}$. The value of $T_{c}$ is found to be closely related to the $e$-ph couplings in the systems; as for the $o F 16-F d d d$ and $h P 3-P 3_{1} 21$ phases, we find that their $\lambda$ decreases significantly with pressure.

To investigate the electronic origins of $o F 16-F d d d$ and $h P 3-P 3_{1} 21$ becoming the energetically preferable phases at pressures above $97 \mathrm{GPa}$, we compare their projected band structures and DOS at 0 and $97 \mathrm{GPa}$ in Fig. 4. It is noticed that the $s$ electrons occupy the low-energy levels at $0 \mathrm{GPa}$, while the $d$ electron energy levels dramatically decrease at $97 \mathrm{GPa}$, which significantly lowers the total energies and results in the transfer of $s$ to $d$ electrons. Similar $s$ to $d$ electron transfer was also concluded from experiments $[29,30]$ and canonical tight-binding calculations [31] for rare earth elements. Therefore, the shift of the $d$ electron energy levels and the related $s$ to $d$ electron transfer are believed to be important for the static stabilities of the $o F 16-F d d d$ and $h P 3-P 3_{1} 21$ phases with increasing pressure. By comparing the DOS at 0 and $97 \mathrm{GPa}$, it is obvious that the electronic states located at low-energy levels, especially those between -4 to $-2 \mathrm{eV}$, increase significantly at $97 \mathrm{GPa}$. In the meantime, the DOS at Fermi level decrease, respectively, from 2.18 and 1.53 (eV atom) ${ }^{-1}$ at $0 \mathrm{GPa}$ to 0.90 and 0.67 (eV atom) $^{-1}$ at $97 \mathrm{GPa}$ for the $o F 16-F d d d$ and $h P 3-P 3_{1} 21$ phases. These considerable changes in DOS are believed to contribute greatly to the phase stabilities of $o F 16-F d d d$ and $h P 3-P 3_{1} 21$ under high pressure.

Charge density differences on the (010) atomic plane of $o F 16-F d d d$ and the (203) atomic plane of $h P 3-P 3_{1} 21$ at 0 and $97 \mathrm{GPa}$ are compared in Fig. 5 in order to investigate the influences of pressure on the chemical bonding of the systems. It is obvious that electrons tend to move away from the $\mathrm{Y} 1-\mathrm{Y} 2$ bond and accumulate between $\mathrm{Y} 3-\mathrm{Y} 4$ for the $o F 16-F d d d$ phase at $97 \mathrm{GPa}$, where the bond lengths of Y1-Y2 and Y3-Y4 are, respectively, 2.63 and $3.27 \AA$. For the $h P 3-P 3_{1} 21$ phase at $97 \mathrm{GPa}$, the electrons move 


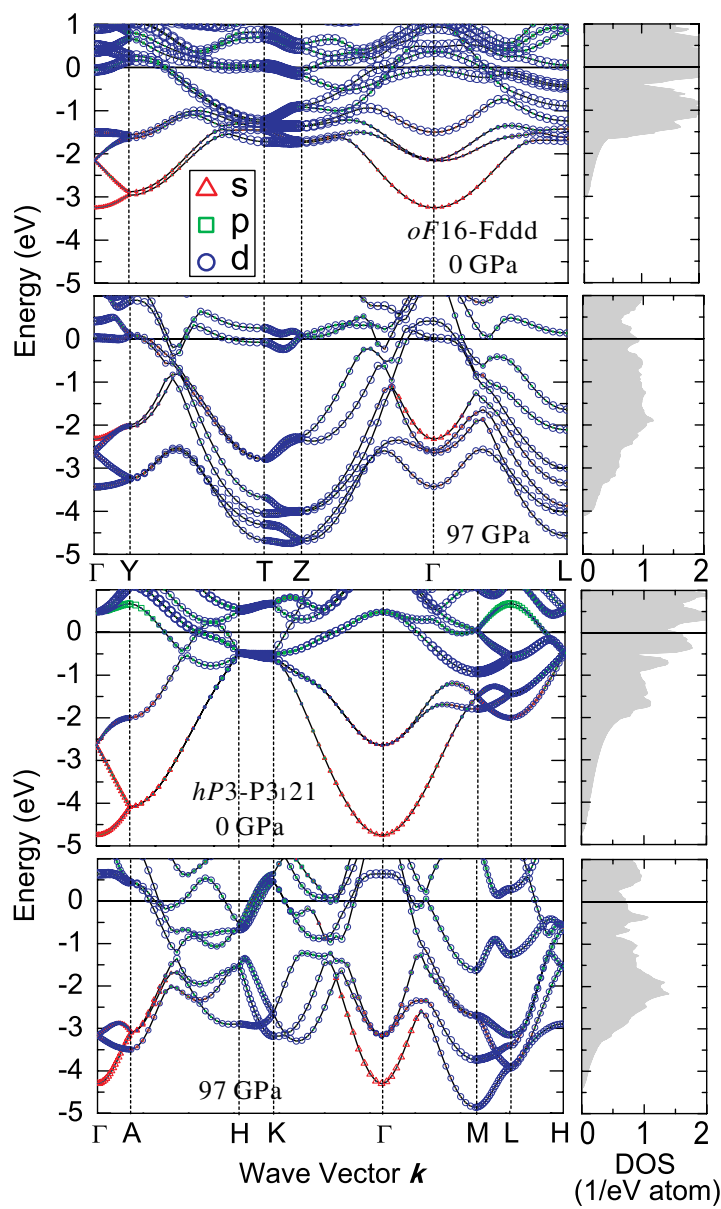

FIG. 4 (color online). The electronic band structures and DOS of the $o F 16-F d d d$ and $h P 3-P 3_{1} 21$ phases under pressures of 0 and $97 \mathrm{GPa}$. Energy is relative to the Fermi level, which is located at $0 \mathrm{eV}$. The projected $s, p$, and $d$ electron characters are denoted by discrete symbols.

away from the Y1-Y2 bond and accumulate between $\mathrm{Y} 1-\mathrm{Y} 4$, nonetheless, no obvious changes in the charge densities are observed for the Y1-Y3 bond. The bond lengths of $\mathrm{Y} 1-\mathrm{Y} 2, \mathrm{Y} 1-\mathrm{Y} 3$, and $\mathrm{Y} 1-\mathrm{Y} 4$ are, respectively, $2.65,2.79$, and $3.10 \AA$ for the $h P 3-P 3_{1} 21$ phase at $97 \mathrm{GPa}$. Therefore, the observed accumulations of electrons to the interstitial sites of the lattices of $o F 16-F d d d$ and $h P 3-P 3_{1} 21$ may account for their stabilities at high pressure. Pickard and Needs have proposed that $s p$-bonded materials could be commonly described by a twocomponent model consisting of positive ions and interstitial electron blobs at multiterapascal pressure [5]. In this Letter, we find that $d$-bonded transition metal $\mathrm{Y}$ already exhibits a tendency of similar behavior at a pressure of $97 \mathrm{GPa}$; thus, the two-component model proposed primarily for $s p$-bonded materials may be valid for more general substances.

In summary, we have employed EAs in conjunction with DFT to perform structure searches for new high-pressure phases of Y metal. The $o F 16-F d d d$ and $h P 3-P 3_{1} 21$ phases
oF16-Fddd

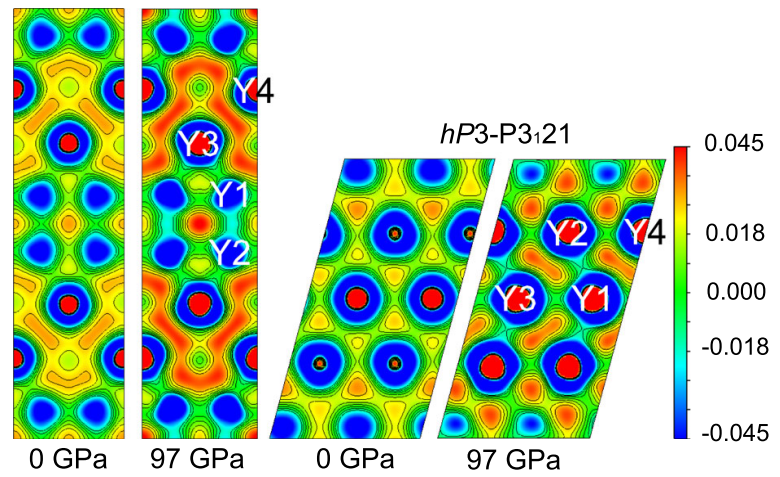

FIG. 5 (color online). The charge density differences (in $\AA^{-3}$, plotted with VESTA [32]) on the (010) atomic plane of $o F 16-F d d d$ and the (203) atomic plane of $h P 3-P 3_{1} 21$ at 0 and $97 \mathrm{GPa}$.

are predicted to be energetically and dynamically stable, and are proposed to be the most probable crystal structures which Y may take in the 97-206 GPa range. We have successfully reproduced the experimentally measured $T_{c}$ of Y below about $100 \mathrm{GPa}$. More importantly, the $T_{c}$ is predicted to decrease under higher pressure, in sharp contrast to its observed behavior below about $100 \mathrm{GPa}$ (it increases monotonically with pressure). The decrease in $T_{c}$ is found to be closely related to the $e$-ph couplings in the systems. By comparing the projected band structures and DOS of the $o F 16-F d d d$ and $h P 3-P 3_{1} 21$ phases at 0 and $97 \mathrm{GPa}$, we find that the shifts of $d$ electron states to lowenergy levels and the significant decreases in the DOS at Fermi levels at high pressure contribute to their phase stabilities. In addition, the accumulations of electrons into the interstitial sites of the lattices also play an important role under high pressure.

Since $\mathrm{Y}$ is one of the highest $T_{c}$ elemental superconductors under high pressure, our proposed new phases and their unexpected behaviors of $T_{c}$ above 97 GPa may stimulate future experimental and theoretical investigations for a better understanding of its superconducting features.

Computations were performed using the facilities of Shenyang Supercomputing Center, Chinese Academy of Sciences (Grant No. INFO-115-B01). The authors acknowledge the financial support from the MoST of China under Grant No. 2011CB606404. Y. C. is also grateful to the T. S. Kê Research Fellowship of IMR/SYNL and the K.C. Wong Education Foundation for financial support.

*Corresponding author: yuechen@columbia.edu

Present address: Department of Applied Physics and Applied Mathematics, Columbia University, New York, New York 10027, USA.

[1] C. J. Pickard and R. J. Needs, Phys. Rev. Lett. 107, 087201 (2011). 
[2] M. Marqués, M.I. McMahon, E. Gregoryanz, M. Hanfland, C. L. Guillaume, C. J. Pickard, G. J. Ackland, and R. J. Nelmes, Phys. Rev. Lett. 106, 095502 (2011).

[3] C. J. Pickard and R. J. Needs, Phys. Rev. Lett. 102, 146401 (2009).

[4] J. Sun, D. D. Klug, C. J. Pickard, and R. J. Needs, Phys. Rev. Lett. 106, 145502 (2011).

[5] C. J. Pickard and R. J. Needs, Nature Mater. 9, 624 (2010).

[6] C. J. Pickard and R. J. Needs, Phys. Rev. Lett. 102, 125702 (2009).

[7] Y. M. Ma, M. Eremets, A. R. Oganov, Y. Xie, I. Trojan, S. Medvedev, A. O. Lyakhov, M. Valle, and V. Prakapenka, Nature (London) 458, 182 (2009).

[8] X. D. Wen, L. Hand, V. Labet, T. Yang, R. Hoffmann, N. W. Ashcroft, A. R. Oganov, and A. O. Lyakhov, Proc. Natl. Acad. Sci. U.S.A. 108, 6833 (2011).

[9] A. R. Oganov, Y. M. Ma, Y. Xu, I. Errea, A. Bergara, and A. O. Lyakhov, Proc. Natl. Acad. Sci. U.S.A. 107, 7646 (2010).

[10] Y. Xie, A. R. Oganov, and Y. M. Ma, Phys. Rev. Lett. 104, 177005 (2010).

[11] A. N. Kolmogorov, S. Shah, E. R. Margine, A. F. Bialon, T. Hammerschmidt, and R. Drautz, Phys. Rev. Lett. 105, 217003 (2010).

[12] K. Shimizu, H. Ishikawa, D. Takao, T. Yagi, and K. Amaya, Nature (London) 419, 597 (2002).

[13] J. J. Hamlin, V. G. Tissen, and J. S. Schilling, Phys. Rev. B 73, 094522 (2006).

[14] S. Lei, D. A. Papaconstantopoulos, and M. J. Mehl, Phys. Rev. B 75, 024512 (2007).

[15] Z.P. Yin, S. Y. Savrasov, and W. E. Pickett, Phys. Rev. B 74, 094519 (2006).

[16] Y. Chen, Q. M. Hu, and R. Yang, Phys. Rev. B 84, 132101 (2011).
[17] A. R. Oganov, A. O. Lyakhov, and M. Valle, Acc. Chem. Res. 44, 227 (2011).

[18] A. R. Oganov and C.W. Glass, J. Chem. Phys. 124, 244704 (2006).

[19] C. W. Glass, A. R. Oganov, and N. Hansen, Comput. Phys. Commun. 175, 713 (2006).

[20] G. Kresse and J. Hafner, Phys. Rev. B 47, 558 (1993).

[21] G. Kresse and J. Hafner, Phys. Rev. B 49, 14251 (1994).

[22] P. E. Blöchl, Phys. Rev. B 50, 17953 (1994).

[23] J.P. Perdew and Y. Wang, Phys. Rev. B 45, 13244 (1992).

[24] H. J. Monkhorst and J. D. Pack, Phys. Rev. B 13, 5188 (1976).

[25] P. Giannozzi et al., http://www.quantum-espresso.org.

[26] Ultrasoft pseudopotentials [D. Vanderbilt, Phys. Rev. B 41, 7892 (1990)] are applied with a plane-wave energy cutoff of 30 Ry. $8 \times 8 \times 8$ and $14 \times 14 \times 6 k$ meshes as well as $4 \times 4 \times 4$ and $6 \times 6 \times 4 q$ meshes are employed for calculating the phonon dispersions of $o F 16-F d d d$ and $h P 3-P 3_{1} 21$, respectively. To evaluate the $e$-ph couplings, we apply a Gaussian broadening of 0.04 Ry with $24 \times$ $24 \times 24$ and $36 \times 36 \times 24 k$ meshes for the $o F 16-F d d d$ and $h P 3-P 3_{1} 21$ phases, respectively.

[27] P. B. Allen and R.C. Dynes, Phys. Rev. B 12, 905 (1975).

[28] P. B. Allen, Handbook of Superconductivity, edited by C. P. Poole, Jr. (Academic Press, New York, 1999) pp. 478-483.

[29] Y. K. Vohra, H. Olijnik, W. Grosshans, and W. B. Holzapfel, Phys. Rev. Lett. 47, 1065 (1981).

[30] H. Olijnyk, J. Phys. Condens. Matter 17, 43 (2005).

[31] J. C. Duthie and D. G. Pettifor, Phys. Rev. Lett. 38, 564 (1977).

[32] K. Momma and F. Izumi, J. Appl. Crystallogr. 41, 653 (2008). 